\title{
Call Admission Control for Voice/Data Integration in Broadband Wireless Networks
}

\author{
Majid Ghaderi and Raouf Boutaba \\ School of Computer Science \\ University of Waterloo, Waterloo N2L 3G1, Canada \\ \{ghaderi, rboutaba\}@uwaterloo.ca
}

\begin{abstract}
This paper addresses bandwidth allocation for an integrated voice and data broadband mobile wireless network. Specifically, we propose a new admission control scheme called EFGC, which is an extension of the well-known fractional guard channel scheme proposed for cellular networks supporting voice traffic. The main idea is to use two acceptance ratios, one for voice calls and the other for data calls in order to maintain the proportional service quality for voice and data traffic while guaranteeing a target handoff failure probability for voice calls. We describe two variations of the proposed scheme: EFGC-REST, a conservative approach which aims at preserving the proportional service quality by sacrificing the bandwidth utilization; and EFGC-UTIL, a greedy approach which achieves higher bandwidth utilization at the expense of increasing the handoff failure probability for voice calls. Simulation results show that our schemes satisfy the hard constraints on handoff failure probability and service differentiation while maintaining a high bandwidth utilization.
\end{abstract}

\section{Introduction}

With the expansion of wireless bandwidth, the next generations of mobile cellular networks are expected to support diverse applications such as voice, data and multimedia, demanding different quality of service (QoS) and bandwidth [1,2]. The bandwidth of the wireless links is inherently limited and is generally much smaller than that of wireline access links. Particularly, for integrated voice/data mobile networks it is necessary to develop mechanisms that can provide effective bandwidth management while satisfying the QoS requirements of both types of traffic.

Bandwidth allocation has been extensively studied in single-service (voice) wireless cellular networks. Hong and Rappaport [3] analyzed the famous guard channel (GC) scheme, which is optimal for minimizing an objective function of call blocking and dropping probabilities. Ramjee et al.[4] proposed a fractional guard channel scheme (FGC) which is optimal for minimizing call blocking probability subject to a hard constraint on call dropping probability. Instead of explicit bandwidth reservation as in GC, the FGC accepts new calls according to a randomization parameter called the acceptance ratio. One advantage of FGC 
over the GC is that it distributes the new accepted calls evenly over time which leads to a more stable control [5].

One of the challenges in moving to a multi-service system is that the limited bandwidth has to be shared among multiple traffics [2]. Epstein and Schwartz [6] investigated complete sharing, complete partitioning and hybrid reservation schemes for two classes of traffic, namely narrow-band and wide-band traffic. Haung et al.[1] proposed a bandwidth allocation scheme for voice/data integration based on the idea of movable boundaries (MB), however they completely neglected the prioritization of handoff calls over new calls and treated the two identically. Wu et al.[2] proposed a dual threshold reservation (DTR) scheme, which is static, i.e., the two reservation thresholds are fixed over time and it is too difficult to adjust them dynamically to satisfy the required service quality. Interested readers are referred to [7] for a comparison between DTR and MB schemes.

This paper introduces an extended fractional guard channel call admission mechanism (EFGC) for integrated voice/data mobile cellular networks that maximizes the wireless bandwidth utilization while satisfying a target call dropping probability and a relative voice/data service differentiation. The main idea is to use two acceptance ratios for voice and data according to the desired dropping probability of voice calls and relative priority of voice calls over data calls. Similar to $[2,6,8]$, we assume that call dropping is not an important issue for data calls and treat handoff and new data calls in the same way. We define the extended MINBLOCK [4] problem as follows:

for a given cell capacity, maximize the bandwidth utilization subject to a hard constraint on the voice call dropping probability and relative voice/data call blocking probability.

To the best of our knowledge, extending the basic fractional guard channel scheme to address the extended MINBLOCK problem is a novel work. We follow an approach similar to the stable admission control algorithm proposed by Wu et al.[5] to derive the acceptance ratios for voice and data. In particular,

1. EFGC is dynamic, therefore, adopts to a wide range of system parameters and traffic conditions.

2. EFGC uses separate acceptance ratios for voice and data calls, therefore, it is very straightforward to enforce a relative or even strict service differentiation between voice and data traffic.

3. The control mechanism is stochastic and periodical to reduce the overhead associated with DCAC-based schemes.

The rest of the paper is organized as follows. Our system model, assumptions and notations are described in section 2. Section 3 is dedicated to the proposed admission control algorithm and presents the details of the analysis of the algorithm. Some numerical results and their analysis are presented in section 4 . Finally, section 5 concludes this paper. 


\section{System Model}

A cellular system which carries both voice and data traffic is considered. Let $\mathrm{BU}$ denote the smallest bandwidth unit that can be allocated to a call $[2,6,8]$. We assume that each voice call requires $b_{v}$ BUs and each data call requires $b_{d}$ BUs for the whole duration of the call. In this system, voice handoff calls have the highest priority then new voice calls are considered and lastly the new and handoff data calls.

Let random variables $d$ and $s$ denote the call duration and cell residency time of call $X$ respectively. Following the convention (as in $[1,2,3,4,5,6,7,8]$ ), we assume that $d$ and $s$ have exponential distribution. In the real word, the cell residence time distribution may not be exponential but exponential distributions provide the mean value analysis, which indicates the performance trend of the system. Furthermore, the proposed admission control algorithm has a periodic control in which the length of the control period is set to less than the average cell residency time of a call in a cell to make the algorithm insensitive to this assumption.

The system under consideration is not necessarily uniform, i.e., each cell can experience a different load. Let $k=\{v, d\}$ denote the type of traffic, i.e., $k=v$ for voice and $k=d$ for data traffic. Below is the notation which will be used throughout this paper.

- $\Theta_{i}$ : the set of the adjacent cells of cell $i$

- $C_{i}$ : the capacity of cell $i$ in terms of BUs

$-P_{d}$ : voice call dropping probability

$-P_{f}$ : voice handoff failure probability

- $P_{Q o S}:$ target voice handoff failure probability

- $\lambda_{i}^{k}$ : type- $k$ new call arrival rate into cell $i$

$-1 / \mu_{k}$ : type- $k$ average call duration

$-1 / h_{k}$ : type- $k$ average cell residency

$-r_{j i}$ : routing probability from cell $j \in \Theta_{i}$ to cell $i$

- $a_{i}^{k}$ : type- $k$ call acceptance ratio of cell $i$

- $E[z]:$ the mean of random variable $z$

$-V[z]$ : the variance of random variable $z$

Our calculations in this paper are based on the handoff failure probability $P_{f}$, which can be related to the call dropping probability $P_{d}$ by the following relation (refer to [3] for more details):

$$
P_{f}=\frac{P_{d}}{1-P_{d}}\left(\frac{\mu_{v}}{h_{v}}\right) .
$$

Notice that exponential assumption is a necessary condition in deriving (1). Please refer to [9] for the handoff probability under a general call duration and cell residency distribution.

Next, we compute all the required probabilities for the rest of our discussion. Let $P_{h}^{k}(t)$ denote the probability that a type- $k$ call hands off before time $t$ and 
remains active until $t$, given that it has been active at time $t=0$. Also, let $P_{s}^{k}(t)$ denote the probability that a type- $k$ call remains active in its home cell until time $t$, given that it has been active at time $t=0$.

$$
\begin{aligned}
& P_{h}^{k}(t)=\operatorname{Pr}\left(s_{k} \leq t\right) \operatorname{Pr}\left(d_{k}>t\right)=\left(1-e^{-h_{k} t}\right) e^{-\mu_{k} t} \\
& P_{s}^{k}(t)=\operatorname{Pr}\left(s_{k}>t\right) \operatorname{Pr}\left(d_{k}>t\right)=e^{-\left(\mu_{k}+h_{k}\right) t}
\end{aligned}
$$

On average, for any call which arrives at time $t^{\prime} \in(0, t]$, we can compute these probabilities as follows.

$$
\begin{aligned}
& \hat{P}_{h}^{k}(t)=\frac{1}{t} \int_{0}^{t} P_{h}^{k}\left(t-t^{\prime}\right) d t^{\prime} \\
& \hat{P}_{s}^{k}(t)=\frac{1}{t} \int_{0}^{t} P_{s}^{k}\left(t-t^{\prime}\right) d t^{\prime}
\end{aligned}
$$

Finally, let $P_{j i}^{k}(t)$ and $\hat{P}_{j i}^{k}(t)$ denote the probability that an active call in cell $j$ at time $t=0$ will be in cell $i$ at time $t$, where $j \in \Theta_{i}$,

$$
\begin{aligned}
P_{j i}^{v}(t) & =P_{h}^{v}(t) h_{j i} \\
\hat{P}_{j i}^{v}(t) & =\hat{P}_{h}^{v}(t) h_{j i} \\
P_{j i}^{d}(t) & =a_{i}^{d}\left[P_{h}^{d}(t) h_{j i}\right] \\
\hat{P}_{j i}^{d}(t) & =a_{i}^{d}\left[\hat{P}_{h}^{d}(t) h_{j i}\right] .
\end{aligned}
$$

In the next section we will use these probabilities to compute the maximal acceptance ratios for voice and data calls with respect to the specified call dropping probability and relative voice/data call blocking probability.

\section{Admission Control Algorithm}

Fig. 1 shows the state transition diagram of the EFGC scheme (call completions/handoffs are not shown). With the exception of state $C$, voice handoffs are always accepted with probability 1 . In state $C$, the system is full and no more calls can be accepted. At each state there are two acceptance ratios for voice and data (one for voice and the other for data). Typically there is a service differentiation (priority) between voice and data calls which governs the relation between these two acceptance ratios. In this study, we assume that this relation is given and EFGC should maintain it given any traffic condition.

It is too expensive and impractical to repeat all the calculations necessary to determine the appropriate $a=\left(a_{v}, a_{d}\right)$ at each system state. Instead, our algorithm has a periodical control structure. At the beginning of each control interval of length $T$, each cell receives some information from its direct neighbor cells. Using this information and QoS constraints (handoff dropping probability and service differentiation), it will calculate an acceptance ratio $a=\left(a_{v}, a_{d}\right)$ for the current control interval. In addition to this, the call blocking probability in 

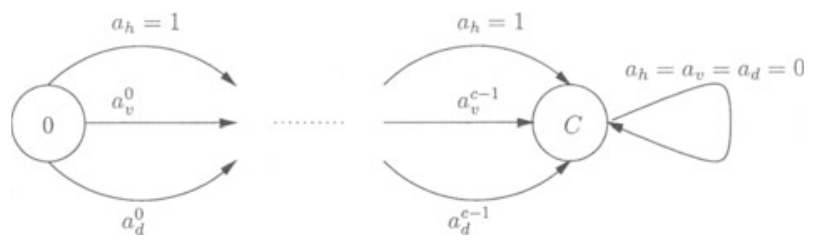

Fig. 1. Extended fractional guard channel.

cell $k$ can be calculated by the acceptance ratio over multiple control periods as follows:

$$
P_{b}^{k}=1-E\left[a_{k}\right]
$$

Therefore, we assume that the ratio of the voice blocking probability to the data blocking probability is given in terms of the relative acceptance probability of voice and data.

The admission control algorithm follows the pseudo-code in Fig. 2. In this algorithm, $X$ is a type- $k$ call where $b(X)$ and $a_{i}(X)$ denote the bandwidth requirement and corresponding acceptance ratios respectively. Also, $C_{i}^{f}(t)$ returns the amount of free bandwidth in cell $i$ at time $t$ and $\operatorname{rand}(0,1)$ is the standard normal random generator function. In the next section, we will specify the acceptance ratio vector $a=\left(a_{v}, a_{d}\right)$ to complete the algorithm.

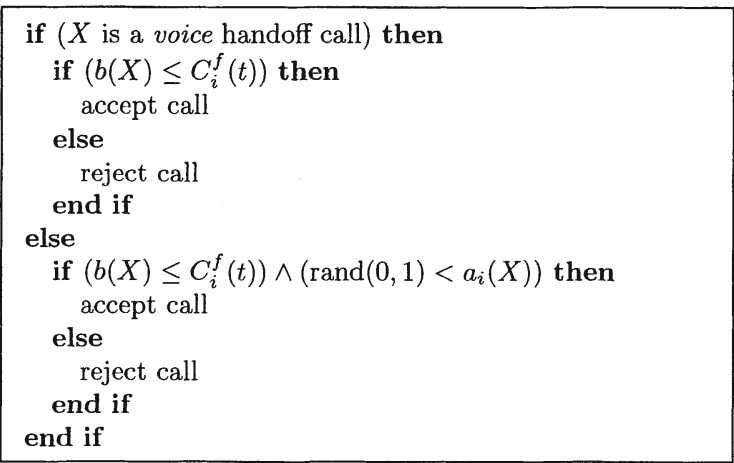

Fig. 2. Call admission control algorithm in cell $i$.

\subsection{Control Algorithm}

We assume that during a control period each call experiences at most one handoff. This assumption is justified by choosing the length of control period $T$ reasonably shorter than the average cell residency time (approximate equations are derived in [10] for appropriate control period length). Therefore, the immediate 
neighbors of cell $i$, i.e., $\Theta_{i}$, are those which will affect the number of calls and consequently the bandwidth usage in cell $i$ during a control period.

The number of calls in cell $i$ at time $t$ is composed of two elements: (1) the number of background calls which are already in cell $i$ or its adjacent cells, and (2) the number of new calls which will arrive in cell $i$ and its adjacent cells during the period $(0, t](0<t \leq T)$. Let $g_{i}^{k}(t)$ and $n_{i}^{k}(t)$ denote the number of background and new type- $k$ calls in cell $i$ at time $t$ respectively. Background calls have a Binomial distribution but new calls have a joint Poisson-Binomial distribution [11]. To have a closed form formula, we approximate this joint distribution by a Binomial distribution.

We then define the following quantities:

$$
\begin{aligned}
V_{s}^{k}(t) & =P_{s}^{k}(t)\left(1-P_{s}^{k}(t)\right) \\
V_{j i}^{k}(t) & =P_{j i}^{k}(t)\left(1-P_{j i}^{k}(t)\right) \\
\hat{V}_{s}^{k}(t) & =\hat{P}_{s}^{k}(t)\left(1-\hat{P}_{s}^{k}(t)\right) \\
\hat{V}_{j i}^{k}(t) & =\hat{P}_{j i}^{k}(t)\left(1-\hat{P}_{j i}^{k}(t)\right)
\end{aligned}
$$

which show the variance of handoff/stay probabilities and are obtained with respect to the properties of Binomial distribution. Then the mean number of type- $k$ active calls in cell $i$ at time $t$ is given by

$$
E\left[N_{i}^{k}(t)\right]=E\left[g_{i}^{k}(t)\right]+E\left[n_{i}^{k}(t)\right]
$$

where,

$$
\begin{aligned}
& E\left[g_{i}^{k}(t)\right]=N_{i}^{k}(0) P_{s}^{k}(t)+\sum_{j \in \Theta_{i}} N_{j}^{k}(0) P_{j i}^{k}(t) \\
& E\left[n_{i}^{k}(t)\right]=\left(a_{i}^{k} \lambda_{i}^{k} t\right) \hat{P}_{s}^{k}(t)+\sum_{j \in \Theta_{i}}\left(a_{j}^{k} \lambda_{j}^{k} t\right) \hat{P}_{j i}^{k}(t)
\end{aligned}
$$

and similarly the variance is

$$
V\left[N_{i}^{k}(t)\right]=V\left[g_{i}^{k}(t)\right]+V\left[n_{i}^{k}(t)\right]
$$

where,

$$
\begin{aligned}
V\left[g_{i}^{k}(t)\right] & =N_{i}^{k}(0) V_{s}^{k}(t)+\sum_{j \in \Theta_{i}} N_{j}^{k}(0) V_{j i}^{k}(t) \\
V\left[n_{i}^{k}(t)\right] & =\left(a_{i}^{k} \lambda_{i}^{k} t\right) \hat{V}_{s}^{k}(t)+\sum_{j \in \Theta_{i}}\left(a_{j}^{k} \lambda_{j}^{k} t\right) \hat{V}_{j i}^{k}(t) .
\end{aligned}
$$

Knowing the bandwidth requirement of each type of calls, the mean and variance of bandwidth usage in cell $i$ at time $t, C_{i}^{u}(t)$, are given by

$$
\begin{aligned}
& E\left[C_{i}^{u}(t)\right]=b_{v} E\left[N_{i}^{v}(t)\right]+b_{d} E\left[N_{i}^{d}(t)\right] \\
& V\left[C_{i}^{u}(t)\right]=b_{v}^{2} V\left[N_{i}^{v}(t)\right]+b_{d}^{2} V\left[N_{i}^{d}(t)\right] .
\end{aligned}
$$

As we mentioned before, the cellular system considered in this paper is a broadband wireless system with capacity of several Mbps. In practice, 3G systems and beyond can be considered as broadband wireless systems (for example 
a UMTS system can support up to $2 \mathrm{Mbps}$ ). With this range of cell capacity it is reasonable to apply the central limit theorem. Then, the bandwidth usage in each cell can be approximated by a normal distribution:

$$
C_{i}^{u}(t) \approx \mathcal{N}\left(E\left[C_{i}^{u}(t)\right], V\left[C_{i}^{u}(t)\right]\right)
$$

Therefore, the original problem of maintaining a target handoff failure probability $P_{f}$ is reduced to maintaining the bandwidth usage below the available capacity $C_{i}$ at any point in time $t \in(0, T]$, or equivalently maintaining the overload probability below $P_{f}$. Hence, the time-dependent handoff failure probability $P_{f}(t)$ can be computed as follows:

$$
P_{f}(t)=\operatorname{Pr}\left(C_{i}^{u}(t)>C_{i}\right)
$$

therefore,

$$
P_{f}(t)=\frac{1}{2} \operatorname{erfc}\left(\frac{C_{i}-E\left[C_{i}^{u}(t)\right]}{\sqrt{2 V\left[C_{i}^{u}(t)\right]}}\right)
$$

where $\operatorname{erfc}(c)$ is the complementary error function. Then, similar to [5], the average handoff failure probability over a control period is given by

$$
\widehat{P}_{f}=\frac{1}{T} \int_{0}^{T} P_{f}(t) d t .
$$

To solve (26) for $a=\left(a_{v}, a_{d}\right)$ we need one more equation. This equation can be derived with respect to the required service differentiation. Given the service condition $a_{d}=\psi\left(a_{v}\right)$, the acceptance ratio vector $a=\left(a_{v}, a_{d}\right)$ can be found by numerically solving (26). Function $\psi$ is such that $0 \leq \psi\left(a_{v}\right) \leq 1$ and $\psi(0)=0$. In addition to this, $\psi$ is uniformly increasing over $[0,1]$. The boundary condition is that $a \in[0,1] \times[0,1]$, hence if $\widehat{P}_{f}<P_{Q o S}$ than $P_{f}$ for $a_{v}=1$ then $a=(1, \psi(1))$. Similarly, if $\widehat{P}_{f}>P_{Q o S}$ even for $a_{v}=0$, then $a=(0,0)$.

Furthermore, we have implemented two versions of this basic algorithm. EFGC-REST (or simply REST) is a restricted version which aims at satisfying the specified priority function $\psi$ over time. In other words, REST always uses the acceptance ratio $a=\left(a_{v}, \psi\left(a_{v}\right)\right)$ regardless of the congestion situation. It is obvious that in some states of the system it is possible to increase the acceptance ratio of data calls beyond the limit returned by the service differentiation function. For example when the network is not congested (at light traffic loads), we found that by increasing the priority of data traffic the overall utilization of the precious wireless bandwidth is increased while the handoff failure remains almost at the same level. This relaxed version is called EFGC-UTIL (or simply UTIL) due to its greedy approach for maximizing the utilization.

To estimate the control parameters of the EFGC, we simply use an exponentially weighted moving average technique. Each cell $i$ estimates the average new call arrival rate of its neighbors based on their previous acceptance ratio:

$$
\left(a_{j}^{k} \lambda_{j}^{k}\right) \leftarrow(1-\epsilon) N_{j}^{k} / T+\epsilon\left(a_{j}^{k} \lambda_{j}^{k}\right)
$$


where $N_{j}^{k}$ is the number of type- $k$ new calls that were actually accepted in cell $j$ during the previous control period and $\epsilon$ is a weighting factor that should be specified with respect to the sampled observations. In our simulations we found that $\epsilon=0.3$ leads to good estimation of the average new call arrival rate.

\section{Numerical Results}

\subsection{Simulation Parameters}

Simulations were performed on a two-dimensional cellular system consisting of 19 hexagonal cells (see Fig. 3). Opposite sides wrap-around to eliminate the finite size effect.

The common parameters used in the simulation are as follows. All the cells have the same capacity $C=5 \mathrm{Mbps}$, which is equal to $160 \mathrm{BU}$ assuming each $\mathrm{BU}$ is equal to $32 \mathrm{Kbps}$ (encoded voice using $\mathrm{ADPCM}$ requires $32 \mathrm{Kbps}$ ). Target handoff failure probability for voice calls is $P_{f}=0.01, T=20 \mathrm{~s}$ and all the neighboring cells have the same chance to be chosen by a call for handoff, i.e. $h_{j i}=1 / 6$. The system is uniform and the input load is the same for every cell, although EFGC is designed to handle the nonuniform case as well. For each load, simulations were done by averaging over 8 samples, each for 10 hours of traffic. Load distribution between voice and data traffic is fixed over time. At any load, $60 \%$ of the load is due to voice calls and the remaining $40 \%$ is composed of data calls. Table 1 summarizes service and traffic parameters for both traffic types. In this table, priority refers to the relative priority of voice and data calls. It means that new voice calls have higher priority than data calls for the admission control algorithm. In particular, the probability of accepting a new voice call is at least twice the probability of accepting a data call (new/handoff) at any time and any load.

Table 1. Voice and data service parameters.

\begin{tabular}{llllll}
\hline Type & Priority & $1 / \mu(s)$ & $1 / h(s)$ & BU & Load \\
\hline voice & 1 & 180 & 100 & 1 & $60 \%$ \\
data & 0.5 & 1000 & 800 & 2 & $40 \%$ \\
\hline
\end{tabular}

As mentioned earlier, this relative priority can be any service differentiation function. In our simulations, for the sake of simplicity we have chosen a constant service differentiation function.

We have also implemented the double threshold reservation (DTR) [2] scheme for comparison purposes. Since DTR is designed for a static traffic pattern, the handoff failure probability increases rapidly with the network load when the guard channels for handoff are few, but remains too low when the guard channels are many. Here, we choose the two thresholds in such a way that DTR achieves its 
objectives when the network starts to get overloaded. Hence, the voice threshold is set to $155 \mathrm{BUs}$ and the data threshold is set to $151 \mathrm{BUs}$. Using these thresholds at load $2, P_{f}$ and $p=a_{v} / a_{d}$ were found to be 0.01 and 2 respectively.

\subsection{Gaussian Verification}

When the network is not congested and each cell has only a few active calls, it is clear that Gaussian approximation is not good. On the other hand, at light loads the admission algorithm does not require a high precision estimation of the load since there is no congestion in the network. As the load increases the number of active calls in each cell increases rapidly until no more calls can be accepted. Due to the high capacity of a broadband system, it is expected to have enough active calls in each cell so that central limit theorem can be applied.

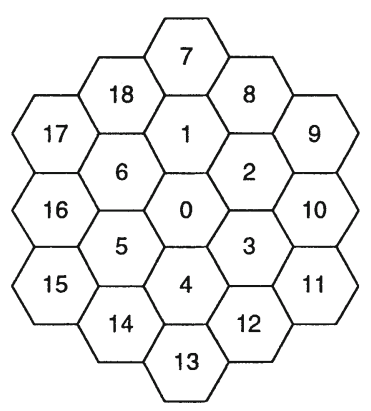

Fig. 3. Simulated cellular system.

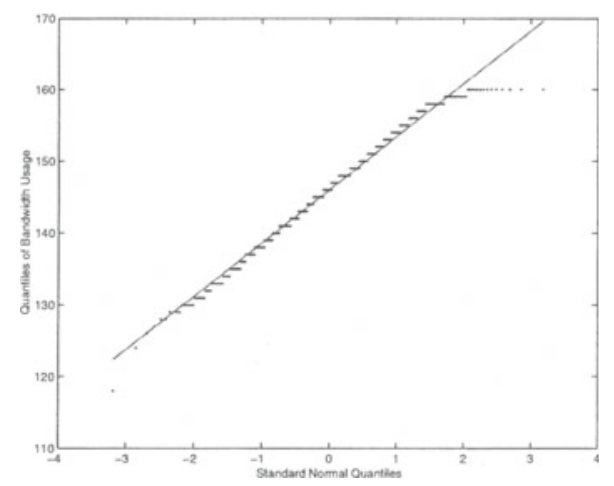

Fig. 4. QQ-plot of bandwidth usage.

We further investigated this issue in our simulation. At the beginning of each interval, the bandwidth usage at cell 0 is recorded until the end of simulation for load 2 (which is not a very high load). To verify the normality of these samples, we used the standard QQ-plot. Fig. 4 depicts the QQ-plot of the sample of the bandwidth usage at cell 0 versus the quantiles of the standard normal distribution. This plot clearly shows that Gaussian approximation of the bandwidth usage in each cell is satisfactory for our stochastic control.

\subsection{Results and Analysis}

Fig. 5 shows the handoff failure probability for the three schemes for a wide range of loads (interested readers are referred to [10] for more detailed simulation results which have not been presented in this paper due to space limitations). Both UTIL and REST maintain a constant failure probability independent of the load. For DTR, it grows very rapidly with the load (which was expected). With light 
loads (load $<2$ ), DTR and REST have almost the same failure probability while UTIL has slightly higher $P_{f}$. But with high loads (load $>2$ ) UTIL and REST are almost matched together while DTR has much higher failure probability. Fig. 8 shows that, although REST has better failure probability in light loads, this is accomplished by substantial sacrifice in the data call blocking probability. In addition to this, even in this region, UTIL has satisfied the target failure probability.

One of the objectives of EFGC is to maintain the relative service priority between voice and data calls. In our simulations, this relative priority is fixed and indicates that the acceptance probability of new voice calls should be twice the acceptance probability of new data calls. Fig. 6 depicts the ratio $p=a_{v} / a_{d}$ for different loads. It shows that EFGC maintains an almost constant service priority between the two types of traffic. Particularly, REST precisely preserves $p=2$ for the whole range of loads while UTIL has $p=1$ in light loads and $p=2$ in high loads as expected because in light loads UTIL accepts as many data calls as there is enough bandwidth (without violating the target voice handoff failure probability). As the load increases, service priority of DTR increases rapidly. Fig. 8 shows that at high loads almost no data call is accepted. In other words, DTR is not fair and leads to starvation of data traffic. We have to mention that, although in this simulation the service differentiation is fixed, the EFGC can satisfy more complex functions such as state dependent functions.
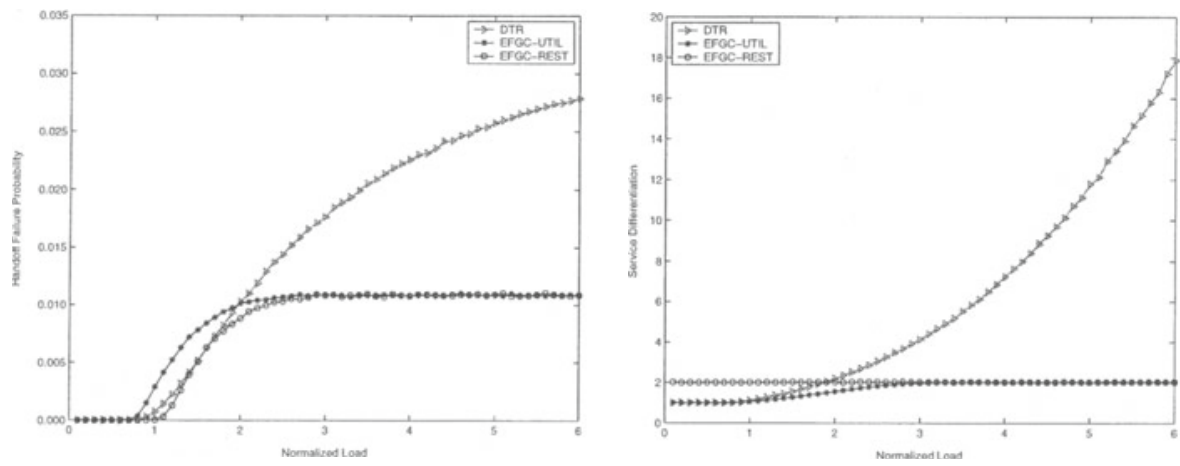

Fig. 5. Voice handoff failure probability. Fig. 6. Relative acceptance probability $(p)$.

Figs. 7 and 8 show the new voice and new/handoff data call acceptance probability respectively. Again for high loads, UTIL and REST converge on the same result but the difference in their performance at light loads is significant. For data traffic at light loads the acceptance probability of UTIL is almost twice that of the REST. This explains why the utilization of UTIL is superior to REST. It can be seen that DTR has slightly higher acceptance probability for voice but much lower acceptance probability for data in comparison to UTIL and REST. 

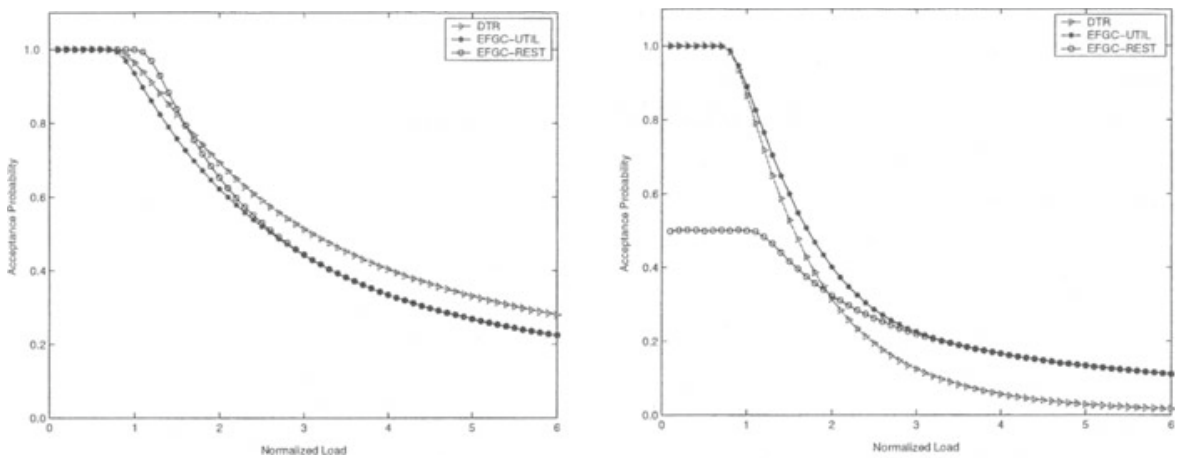

Fig. 7. Voice calls acceptance probability. Fig. 8. Data calls acceptance probability.

And finally, Fig. 9 depicts the wireless bandwidth utilization under the three bandwidth allocation mechanisms. Although DTR performs poorly in terms of handoff failure probability and service priority, its utilization is slightly better than EFGC. Interestingly, UTIL has exactly the same utilization as DTR at light loads and as expected, higher utilization than REST. In this simulation, voice traffic constitutes a larger portion of the total load. As the percentage of data traffic increases, the utilization of DTR is expected to drop (interested readers are referred to [10] for more detailed simulation results).

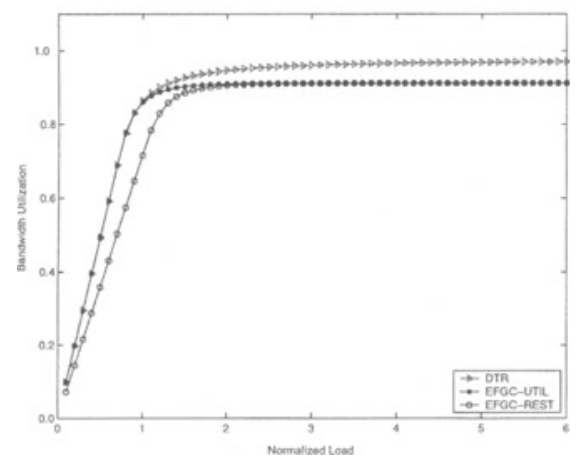

Fig. 9. Wireless bandwidth utilization.

\section{Conclusion}

In this paper, we developed a new admission control algorithm for voice/data integration in broadband wireless networks. Our algorithm is a natural extension of the well-known fractional guard channel proposed for voice cellular systems. EFGC always achieves the predetermined call dropping probability for voice calls while keeping the relative blocking probability of voice and data calls within a 
target threshold. We then described two versions of the EFGC, namely EFGCUTIL and EFGC-REST. EFGC-UTIL follows a greedy approach to maximize the bandwidth utilization while EFGC-REST maintains the relative service priority. Both versions converged on the same result for high traffic loads. The major advantage of EFGC is its insensitivity to the network load. The dropping probability of voice and relative blocking probability of voice and data calls is maintained at a stable level over a wide range of traffic loads. According to the simulation results, we conclude that EFGC-UTIL is a good candidate for integrated voice/data cellular networks.

We are currently investigating the case of multiple classes of traffic where each class has its own QoS requirements in terms of call blocking and dropping probability. EFGC can readily support multiple classes of traffic by assigning a separate acceptance ratio to each class. However, computing these acceptance ratios in order to satisfy the desired QoS is not trivial.

\section{References}

1. Haung, Y.R., Lin, Y.B., Ho, J.M.: Performance analysis for voice/data integration on a finite-buffer mobile system. IEEE Trans. Veh. Technol. 49 (2000) 367-378

2. Wu, H., Li, L., Li, B., Yin, L., Chlamtac, I., Li, B.: On handoff performance for an integrated voice/data cellular system. In: Proc. IEEE PIMRC'02. Volume 5., Lisboa, Portugal (2002) 2180-2184

3. Hong, D., Rappaport, S.S.: Traffic model and performance analysis for cellular mobile radio telephone systems with prioritized and nonprioritized handoff procedures. IEEE Trans. Veh. Technol. 35 (1986) 77-92

4. Ramjee, R., Towsley, D., Nagarajan, R.: On optimal call admission control in cellular networks. ACM/Baltzer J. Wireless Networks 3 (1997) 29-41

5. Wu, S., Wong, K.Y.M., Li, B.: A dynamic call admission policy with precision QoS guarantee using stochastic control for mobile wireless networks. IEEE/ACM Trans. Networking 10 (2002) 257-271

6. Epstein, B., Schwartz, M.: Reservation strategies for multi-media traffic in a wireless environment. In: Proc. IEEE VTC'95. Volume 1., Chicago, USA (1995) 165169

7. Li, B., Li, L., Li, B., Cao, X.R.: On handoff performance for an integrated voice/data cellular system. ACM/Baltzer J. Wireless Networks 9 (2003) 393-402

8. Li, B., Chanson, S., Lin, C.: Analysis of a hybrid cutoff priority scheme for multiple classes of traffic in multimedia wireless networks. ACM/Baltzer J. Wireless Networks 4 (1998)

9. Fang, Y., Chlamtac, I.: Analytical generalized results for handoff probability in wireless networks. IEEE Trans. Commun. 50 (2002) 396-399

10. Ghaderi, M., Boutaba, R.: Call admission control for voice/data integration in broadband wireless networks. Technical Report CS-2003-41, School of Computer Science, University of Waterloo (2003)

11. Naghshineh, M., Schwartz, M.: Distributed call admission control in mobile/wireless networks. IEEE J. Select. Areas Commun. 14 (1996) 711-717 